\section{Analysis of the sulphite content in shrimps and prawns}

\author{
Elisabetta Bonerba, Edmondo Ceci, \\ Giancarlo Bozzo, Angela Di Pinto, \\ Giuseppina Tantillo \\ Dipartimento di Sanità Pubblica e \\ Zootecnia, Facoltà di Medicina \\ Veterinaria, Università degli Studi di Bari \\ Aldo Moro, Valenzano, Italy
}

\section{Abstract}

Food additives are redefined in European legislation (EC Regulation No. 1333/2008). Sulphur dioxide (E220) and sulphite (E221E228) are widely used in food processing as preservatives because they slow down bacterial growth on foods and prevent oxidation or browning developing on shrimp and lobster.

Shellfish processors, farmers and fishermen have long used sulfiting agents in a variety of species of warm and coldwater crustaceans as a treatment to prevent prawns and shrimps melanosis (blackspot), which is a natural process that makes the shell black after harvesting caused by Polyphenoloxidase enzyme systems which remain active during refrigeration or ice storage. Sulfite-induced hypersensitivity is the most well-established adverse response in humans to this food additive. In the present study the presence of sulfites in different frozen and thawed shrimp and prawn species belonging to Penaeoidea superfamily has been evaluated by the Monier-Williams procedure, in order to carry out a risk assessment and evaluate the levels of consumer exposure to this class of additives from these fish products. In addition to assessing and monitoring the correct use of the additive, according to the limits imposed by the European regulations, the correct consumer information on labels was also evaluated. Analysis were performed on both whole shrimp (shell on) and inedible parts (head and peeled shell). Sulphites concentration in frozen samples (expressed as $\mathrm{SO}_{2} \mathrm{mg} / \mathrm{kg}$ mean value \pm S.D.) was $214 \pm 17.43$ for head on shell on shrimps; $170.73 \pm 14.99$ for shell on headless shrimps; $112.90 \pm 27.55$ for peeled and deveined shrimps. Thawed shrimps were purchased at mass retailers channel and local fish markets and local seafood retailers and purveyors: for these samples, all head on shell on, the sulphites concentration (expressed as $\mathrm{SO}_{2} \mathrm{mg} / \mathrm{kg}$ mean value \pm S.D.) was $160.05 \pm 26.15$ and $292.54 \pm 146.04$, respectively. Non-edible parts showed, in all samples, much higher concentrations.

\section{Introduzione}

L'anidride solforosa è stata utilizzata sin dall'antichità per le sue proprietà disinfettanti $\mathrm{e}$ ancora oggi rappresenta uno degli additivi più impiegati nell'industria alimentare come conservante per alimenti di origine vegetale e animale (Pearson, 1976).

Dal punto di vista chimico-fisico l'anidride solforosa è un gas incolore, non infiammabile, tossico per inalazione, molto solubile in acqua, con un caratteristico odore di uova marce, con punto di ebollizione di $-10^{\circ} \mathrm{C}$; può essere aggiunta agli alimenti come tale (in forma liquida 0 gassosa) oppure sotto forma di composti derivati, come solfiti, bisolfiti e metabisolfiti ampiamente utilizzati come conservanti per una vasta gamma di bevande e prodotti alimentari (Walker, 1985; Taylor et al., 1986; Ruiter et al., 2005). L'anidride solforosa e i solfiti esplicano una spiccata azione antimicrobica (Taylor et al., 1986; Fazio e Warner, 1990; FDA, 2000; Ruiter e Bergwerff, 2005; Wood et $a l ., 2004)$. Il meccanismo d'azione non è ancora del tutto precisato; si ritiene che si instaurino interazioni tra $\mathrm{i}$ bisolfiti e l'acetaldeide all'interno della cellula microbica, o che avvenga la riduzione dei legami disolfuro presenti in enzimi cellulari con importanti funzioni biologiche e conseguente blocco dell'attività enzimatica del microrganismo, o la formazione di addotti che bloccano i normali meccanismi di respirazione della cellula microbica e che alterano la produzione di nicotinammide adenina dinucleotide (NAD).

L'effetto antimicrobico dei solfiti risulta particolarmente efficace su Gram negativi, e in particolare nei confronti dei batteri lattici, acetici e Enterobatteriacee (Daniel, 1986).

Inoltre l'anidride solforosa e i suoi derivati possono inibire lo sviluppo del cosiddetto imbrunimento sia enzimatico che non (Williams et al., 1990; Ruiz-Capillas e JiménezColmenero 2009); il primo è un processo di ossidazione catalizzato da particolari enzimi, che consentono la formazione e l'accumulo di melanoidine, mentre l'imbrunimento non enzimatico è dato da particolari interazioni chimiche tra aminoacidi e gruppi carbonilici dell'alimento che inducono un graduale accumulo di peculiari sostanze di colore nerastro e di sapore amarognolo. In questi casi l'anidride solforosa riduce tali reazioni, legandosi a composti intermedi come le aldeidi.

Anidride solforosa e solfiti possono essere impiegati a scopo fraudolento con l'intento di nascondere il deterioramento degli alimenti prevenendo l'insorgenza di alterazioni microbiche putrefattive; l'utilizzo, se non indicato in etichetta, e se non vengono rispettate le dosi di impiego previste per gli alimenti, può causare nell'uomo diverse sintomatologie, varie per gravità, ma strettamente correlate alla dose
Correspondence: Elisabetta Bonerba Dipartimento di Sanità Pubblica e Zootecnia, Facoltà di Medicina Veterinaria, Università degli Studi di Bari Aldo Moro, S.P. 172 km 3, 70010 Valenzano, Italy.

Tel. +39.080 .5443850 - Fax. +39.080 .5443855 .

E-mail: elisabetta.bonerba@uniba.it

Key words: Food additives, Sulphite, Shrimps, Prawns, Monier-Williams method.

Conflict of interests: the authors declare no potential conflict of interests.

Received for publication: 15 January 2013

Revision received: 16 April 2013.

Accepted for publication: 16 April 2013

This work is licensed under a Creative Commons Attribution 3.0 License (by-nc 3.0).

(OCopyright E. Bonerba et al., 2013

Licensee PAGEPress, Italy

Italian Journal of Food Safety 2013; 2:e18

doi:10.4081/ijfs.2013.e18

assunta e al tipo di esposizione. Le manifestazioni meno gravi comprendono rinite, eritemi cutanei, congiuntivite, nausea e sintomi da irritazione gastrointestinale, mentre nei casi più gravi possono manifestarsi orticaria generalizzata, broncospasmo, edema della glottide, ipotensione e talora shock anafilattico che può insorgere a breve distanza dall'ingestione del pasto (Vally et al., 2009).

La patogenesi della tossicità dei solfiti non è stata ancora del tutto accertata. Tre sono i possibili meccanismi chiamati in causa: una reazione immunomediata da anticorpi di tipo IgE, una risposta colinergica indotta dai solfiti e la deficienza dell'enzima solfito ossidasi (Lester, 1995; Bush et al., 1986; Haahtela e Hannuksela, 1989).

Tra i derivati dell'anidride solforosa, il metabisolfito di sodio (E 223) è l'additivo più comunemente utilizzato per il trattamento dei prodotti della pesca (Hardisson et al., 2002) e in particolare per i crostacei.

Uno dei principali fattori di deterioramento dei crostacei è la decolorazione ossidativa, ossia l'imbrunimento progressivo delle branchie e dei tessuti fibrosi che formano i legamenti intersegmentari del carapace. Questo processo è chiamato melanosi o black spot. I prodotti ittici che presentano melanosi non sono nocivi per la salute umana, ma il danno viene percepito dal consumatore come una perdita delle qualità organolettiche di freschezza del gambero (Montero et al., 2001).

La melanosi è un processo biochimico che si innesca in presenza di ossigeno per azione di un complesso enzimatico endogeno del gambero, le polifenolossidasi (PPO), che convertono i monofenoli (incolori) in difenoli (Bartolo 
e Birk, 1998; Mcevily et al., 1991). Le PPO catalizzano la idrossilazione dell'0-diidrossifenolo a benzochinone, e questo, per autossidazione, reagisce con una varietà di composti quali ammine e amminoacidi, che polimerizzandosi danno origine alla melanina che si presenta sotto forma di pigmenti scuri, insolubili e ad alto peso molecolare (Otwell e Marshall, 1986; Garcia-Molina et al., 2005) che si localizzano prevalentemente a livello di carapace, cefalotorace, esoscheletro della porzione addominale, pleopodi e telson (Zamorano et al., 2009).

La melanosi può essere controllata con varie tecniche, ma l'utilizzo dei solfiti rimane quella che offre i risultati migliori e duraturi, perché interviene direttamente sul processo di ossidazione inibendo l'attività delle PPO.

Dopo la cattura dei crostacei, i solfiti, e in particolare del metabisolfito, sono impiegati con diverse modalità. Quella più diffusa prevede il raffreddamento rapido del pescato, immergendolo in una soluzione acquosa al 5$8 \%$ di metabisolfito e mantenendo la temperatura prossima a $0^{\circ} \mathrm{C}$ per un periodo compreso tra i 10 e i 15 min (Rotllant et al., 2002). Tale impiego presenta alcune criticità: i) la concentrazione di sodio metabisolfito e la durata di immersione devono essere adattati alle dimen- sioni dei crostacei; un gambero di piccola dimensione assorbe il prodotto molto più velocemente. Il tempo di immersione deve essere rigorosamente rispettato, pertanto i piani di autocontrollo e in particolare i diagrammi di flusso relativi al processamento dei gamberi devono essere ben implementati e seguiti; ii) l'immersione nella soluzione di bisolfito deve avvenire contestualmente alla preparazione della stessa, per evitare perdite di concentrazione e diminuzione del titolo della soluzione di metabisolfito dovute all'ossidazione con l'aria; iii) la concentrazione di metabisolfito diminuisce con l'immersione del pescato nella

Tabella 1. Concentrazione di solfiti [espressa in $\left(\mathrm{SO}_{2}\right) \mathrm{mg} / \mathrm{kg}$ ] rilevata nei campioni di gamberi surgelati.

\begin{tabular}{|c|c|c|c|c|}
\hline $\begin{array}{l}\text { Campioni surgelati }(\mathrm{n}=45) \\
\text { Provenienza zona FAO }\end{array}$ & Specie & Tipologia prodotto & Tal quale & Esoscheletro \\
\hline Area 21 - Oceano Atlantico Nord-Ovest & Pandalus borealis & Sgusciato & 85,21 & - \\
\hline Area 27 - Oceano Atlantico Nord-Est & Pandalus borealis & Sgusciato & 78,27 & - \\
\hline Area 27 - Oceano Atlantico Nord-Est & Paeneus kerathurus & Sgusciato & 64,72 & - \\
\hline Area 31 - Oceano Atlantico Centro-Ovest & Penaeus vannamei & Sgusciato & 89,66 & - \\
\hline Area 34 - Oceano Atlantico Centro-Est & Pandalus borealis & Sgusciato & 96,32 & - \\
\hline Area 41 - Oceano Atlantico Sud-Ovest & Paeneus kerathurus & Sgusciato & 103,26 & - \\
\hline Area 51 - Oceano Indiano Ovest & Penaeus monodon & Sgusciato & 133,62 & - \\
\hline Area 57 - Oceano Indiano Est & Penaeus monodon & Sgusciato & 134,87 & - \\
\hline Area 58 - Oceano Indiano Antartico e Sud & Pleoticus muelleri & Sgusciato & 141,45 & - \\
\hline Area 58 - Oceano Indiano Antartico e Sud & Paeneus kerathurus & Sgusciato & 140,64 & - \\
\hline Area 61 - Oceano Pacifico Nord-Ovest & Penaeus monodon & Sgusciato & 135,23 & - \\
\hline Area 67 - Oceano Pacifico Nord-Est & Penaeus vannamei & Sgusciato & 149,44 & - \\
\hline Area 71 - Oceano Pacifico Centro-Ovest & Paeneus indicus & Sgusciato & 94,78 & - \\
\hline Area 77 - Oceano Pacifico Centro-Est & Penaeus vannamei & Sgusciato & 106,14 & - \\
\hline Area 87 - Oceano Pacifico Sud-Est & Penaeus longirostris & Sgusciato & 139,93 & - \\
\hline Area 21 - Oceano Atlantico Nord-Ovest & Penaeus vannamei & Intero & 210,36 & 1335,60 \\
\hline Area 27 - Oceano Atlantico Nord-Est & Pleoticus muelleri & Intero & 201,60 & 1364,40 \\
\hline Area 31 - Oceano Atlantico Centro-Ovest & Penaeus vannamei & Intero & 231,60 & 1531,20 \\
\hline Area 34 - Oceano Atlantico Centro-Est & Penaeus longirostris & Intero & 214,84 & 1324,20 \\
\hline Area 41 - Oceano Atlantico Sud-Ovest & Penaeus vannamei & Intero & 223,59 & 1674,15 \\
\hline Area 47 - Oceano Atlantico Sud-Est & Penaeus monodon & Intero & 206,50 & 1862,48 \\
\hline Area 51 - Oceano Indiano Ovest & Paeneus indicus & Intero & 228,60 & 1614,48 \\
\hline Area 57 - Oceano Indiano Est & Pleoticus muelleri & Intero & 265,47 & 1987,21 \\
\hline Area 58 - Oceano Indiano Antartico e Sud & Paeneus kerathurus & Intero & 202,61 & 1397,13 \\
\hline Area 61 - Oceano Pacifico Nord-Ovest & Pandalus borealis & Intero & 209,45 & 1456,14 \\
\hline Area 67 - Oceano Pacifico Nord-Est & Penaeus monodon & Intero & 203,47 & 1335,45 \\
\hline Area 71 - Oceano Pacifico Centro-Ovest & Penaeus monodon & Intero & 206,93 & 1338,31 \\
\hline Area 77 - Oceano Pacifico Centro-Est & Paeneus kerathurus & Intero & 201,54 & 1331,96 \\
\hline Area 81 - Oceano Pacifico Sud-Ovest & Penaeus longirostris & Intero & 202,38 & 1326,85 \\
\hline Area 87 - Oceano Pacifico Sud-Est & Pandalus borealis & Intero & 201,16 & 1334,12 \\
\hline Area 21 - Oceano Atlantico Nord-Ovest & Penaeus longirostris & Code con guscio & 174,96 & 1710 \\
\hline Area 27 - Oceano Atlantico Nord-Est & Penaeus monodon & Code con guscio & 162,08 & 1683,62 \\
\hline Area 31 - Oceano Atlantico Centro-Ovest & Penaeus vannamei & Code con guscio & 194,78 & 1590 \\
\hline Area 34 - Oceano Atlantico Centro-Est & Pleoticus muelleri & Code con guscio & 186 & 1528,8 \\
\hline Area 41 - Oceano Atlantico Sud-Ovest & Paeneus indicus & Code con guscio & 157,60 & 1503,6 \\
\hline Area 47 - Oceano Atlantico Sud-Est & Paeneus kerathurus & Code con guscio & 202,83 & 1683,6 \\
\hline Area 51 - Oceano Indiano Ovest & Paeneus indicus & Code con guscio & 164,31 & 1695,45 \\
\hline Area 57 - Oceano Indiano Est & Paeneus indicus & Code con guscio & 161,34 & 1646,38 \\
\hline Area 58 - Oceano Indiano Antartico e Sud & Penaeus vannamei & Code con guscio & 156,87 & 1598,26 \\
\hline Area 61 - Oceano Pacifico Nord-Ovest & Penaeus longirostris & Code con guscio & 152,14 & 1586,49 \\
\hline Area 67 - Oceano Pacifico Nord-Est & Paeneus kerathurus & Code con guscio & 155,74 & 1601,35 \\
\hline Area 71 - Oceano Pacifico Centro-Ovest & Penaeus longirostris & Code con guscio & 181,23 & 1985,64 \\
\hline Area 77 - Oceano Pacifico Centro-Est & Penaeus monodon & Code con guscio & 165,54 & 1605,20 \\
\hline Area 81 - Oceano Pacifico Sud-Ovest & Pandalus borealis & Code con guscio & 171,32 & 1784,21 \\
\hline Area 87 - Oceano Pacifico Sud-Est & Penaeus monodon & Code con guscio & 174,35 & 1703,56 \\
\hline
\end{tabular}

FAO, Food and Agriculture Organization 
Tabella 2. Concentrazione di solfiti [espressa in $\left(\mathrm{SO}_{2}\right) \mathrm{mg} / \mathrm{kg}$ ] rilevata nei campioni di gamberi decongelati.

\begin{tabular}{|c|c|c|c|c|c|}
\hline $\begin{array}{l}\text { Campioni decongelati }(\mathrm{n}=45) \\
\text { Provenienza zona FAO }\end{array}$ & Specie & Punto vendita & Tal quale & {$\left[\mathrm{SO}_{2}\right] \mathrm{mg} / \mathrm{kg}$} & $\begin{array}{l}\text { Solfiti in } \\
\text { etichetta }\end{array}$ \\
\hline Area 51 - Oceano Indiano Ovest & Paeneus kerathurus & GDO & 185,26 & 1245,80 & SI \\
\hline Area 57 - Oceano Indiano Est & Penaeus vannamei & GDO & 169,84 & 1248,65 & SI \\
\hline Area 31 - Oceano Atlantico Centro-Ovest & Penaeus vannamei & GDO & 143,59 & 1461 & SI \\
\hline Area 34 - Oceano Atlantico Centro-Est & Penaeus monodon & GDO & 178,45 & 1253 & SI \\
\hline Area 71 - Oceano Pacifico Centro-Ovest & Penaeus longirostris & GDO & 137 & 1857 & SI \\
\hline Area 77 - Oceano Pacifico Centro-Est & Penaeus monodon & GDO & 195,26 & 1127 & SI \\
\hline Area 51 - Oceano Indiano Ovest & Paeneus indicus & GDO & 114,37 & 777 & SI \\
\hline Area 57 - Oceano Indiano Est & Paeneus indicus & GDO & 185 & 1653,38 & SI \\
\hline Area 61 - Oceano Pacifico Nord-Ovest & Penaeus vannamei & GDO & 110 & 731,45 & SI \\
\hline Area 67 - Oceano Pacifico Nord-Est & Paeneus kerathurus & GDO & 159,55 & 1521,56 & SI \\
\hline Area 71 - Oceano Pacifico Centro-Ovest & Pandalus borealis & GDO & 136,84 & 1124,58 & SI \\
\hline Area 77 - Oceano Pacifico Centro-Est & Pandalus borealis & GDO & 143,72 & 1521 & SI \\
\hline Area 71 - Oceano Pacifico Centro-Ovest & Pandalus borealis & GDO & 138,31 & 1425,32 & SI \\
\hline Area 77 - Oceano Pacifico Centro-Est & Pleoticus muelleri & GDO & 178,65 & 1120 & SI \\
\hline Area 87 - Oceano Pacifico Sud-Est & Pleoticus muelleri & GDO & 167,56 & 980,44 & SI \\
\hline Area 21 - Oceano Atlantico Nord-Ovest & Pleoticus muelleri & GDO & 145,65 & 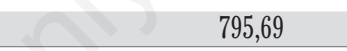 & SI \\
\hline Area 27 - Oceano Atlantico Nord-Est & Penaeus vannamei & GDO & 146,94 & 875,46 & SI \\
\hline Area 31 - Oceano Atlantico Centro-Ovest & Penaeus monodon & GDO & 178,65 & 1025 & SI \\
\hline Area 34 - Oceano Atlantico Centro-Est & Penaeus vannamei & GDO & 198,54 & 1156,64 & SI \\
\hline Area 41 - Oceano Atlantico Sud-Ovest & Penaeus monodon & GDO & 187,98 & 1345,24 & SI \\
\hline$?$ & Penaeus longirostris & MI & 175 & 1445 & NO \\
\hline$?$ & Penaeus Vannamei & MI & 189,84 & 1248 & NO \\
\hline$?$ & Pleoticus muelleri & MI & 144,59 & 1581 & NO \\
\hline$?$ & Penaeus vannamei & MI & 178,95 & 1373 & NO \\
\hline$?$ & Penaeus longirostris & MI & 177 & 1497 & NO \\
\hline$?$ & Penaeus vannamei & MI & 192,23 & 1357 & NO \\
\hline$?$ & Penaeus monodon & MI & 173,37 & 677 & NO \\
\hline$?$ & Paeneus indicus & $\mathrm{P}$ & 194 & 1603,3 & NO \\
\hline$?$ & Paeneus indicus & $P$ & 189 & 737 & NO \\
\hline$?$ & Penaeus vannamei & $\mathrm{P}$ & 167,05 & 1401 & NO \\
\hline$?$ & Penaeus longirostris & $P$ & 624 & 2246 & NO \\
\hline$?$ & Paeneus kerathurus & $\mathrm{P}$ & 310 & 1248 & NO \\
\hline$?$ & Paeneus indicus & $P$ & 123,20 & 598,07 & NO \\
\hline$?$ & Paeneus kerathurus & MI & 557 & 2023 & NO \\
\hline$+a$ & Paeneus indicus & MI & 350 & 1351 & NO \\
\hline 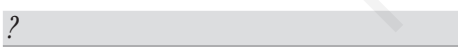 & Pleoticus muelleri & MI & 462 & 1789,06 & NO \\
\hline$?$ & Penaeus longirostris & MI & 421,09 & 1598,02 & NO \\
\hline$?$ & Penaeus longirostris & MI & 223,44 & 1367,78 & NO \\
\hline$?$ & Pandalus borealis & MI & 164,8 & 752,55 & NO \\
\hline$?$ & Pleoticus muelleri & $\mathrm{P}$ & 322,33 & 1398,41 & NO \\
\hline$?$ & Penaeus longirostris & $P$ & 524,61 & 1235,48 & NO \\
\hline$?$ & Pandalus borealis & $\mathrm{P}$ & 458,23 & 965 & NO \\
\hline$?$ & Pleoticus muelleri & $P$ & 421,56 & 1142,26 & NO \\
\hline$?$ & Pandalus borealis & $\mathrm{P}$ & 312,15 & 1158,24 & NO \\
\hline$?$ & Penaeus longirostris & $P$ & 256 & 845 & NO \\
\hline
\end{tabular}

FAO, Food and Agriculture Organization; GDO, grande distribuzione organizzata; ?, provenienza sconosciuta; MI, mercato ittico; P, pescheria. 
soluzione, a causa d'assorbimento da parte del prodotto; in questo caso se gli operatori non sono adeguatamente formati, e non utilizzano metodi rapidi e standardizzati per il controllo della concentrazione della soluzione, sarà difficile ottenere lotti omogenei di gamberi e si correrà il rischio di superare le dosi consentite; iv) la miscela di acqua, ghiaccio e metabisolfito deve essere agitata costantemente, per garantire che tutti i gamberi assorbano l'additivo in modo uniforme; v) il contenuto di $\mathrm{SO}_{2}$ nella soluzione varia a seconda del livello di ossigenazione dell'acqua dato che il metabisolfito è un sequestrante dell'ossigeno; pertanto anche le caratteristiche dell'acqua utilizzata devono essere monitorate; vi) le soluzioni di metabisolfito devono rigorosamente essere preparate in luoghi aperti 0 comunque aerati per evitare eventuali intossicazioni da anidride solforosa e inoltre gli operatori del settore alimentare (OSA) devono indossare dispositivi di protezione individuale (DPI) adeguati quali tute protettive, guanti e maschere antigas.

In realtà lavorative economicamente più piccole l'applicazione del sodio metabisolfito avviene per nebulizzazione di soluzioni preparate grossolanamente 0 addirittura per aspersione della polvere di metabisolfito direttamente sul pescato; tali pratiche risultano rischiose per gli operatori, e non consentono un adeguato controllo sull'effettiva quantità di additivo impiegato.

La nebulizzazione di soluzioni di metabisolfito viene effettuata dopo l'incassettamento dei crostacei. Il pescato viene disposto in strati all'interno di cassette in polistirolo alternando lo stesso con ghiaccio in scaglie; solitamente la nebulizzazione interessa solo lo strato superiore del prodotto, si confida, infatti, nell'effetto diluitivo e di percolamento della soluzione a partire dallo strato più superficiale fino al fondo della cassetta. Anche con questa modalità sono presenti numerose criticità che comportano una additivazione del prodotto spesso non uniforme, e soprattutto non rispettosa dei limiti imposti dalla normativa. Per questi motivi ci è sembrato interessante avviare un'indagine conoscitiva sulla presenza quali-quantitatva di solfiti su gamberi, appartenenti a diverse specie della superfamiglia Penaeoidea, poiché questi prodotti della pesca sono abbondantemente presenti sul mercato, come prodotto fresco, congelato e surgelato, per l'ampia diffusione delle diverse specie riscontrabile in tutti i mari e alle diverse latitudini, e anche per la discreta facilità di adattamento dimostrata alle pratiche di allevamento.

Il Regolamento UE 1129/2011, tra le categorie alimentari riportate, inserisce i gamberi nella classe 09.1.2 Molluschi e crostacei non trasformati, consentendo il possibile utilizzo di solfiti come additivi e indicando il livello massimo (espresso come $\mathrm{mg} / \mathrm{kg}$ di $\mathrm{SO}_{2}$ ) solo nelle parti commestibili di tali prodotti freschi, con- gelati e surgelati in considerazione del numero di unità di vendita: $<80$ unità $\mathrm{SO}_{2}$ $\mathrm{mg} / \mathrm{kg}=150 ; 80 \leq 120$ unità $\mathrm{SO}_{2} \mathrm{mg} / \mathrm{kg}=200 ;$ $>120$ unità $\mathrm{SO}_{2} \mathrm{mg} / \mathrm{kg}=300$.

Pertanto, quanto disciplinato da tale regolamento, risulta applicabile solo alla parte edibile dei crostacei; tuttavia la maggior aliquota commerciale dei gamberi presenti sul mercato viene venduta come gambero intero o comunque provvisto di esoscheletro e nessuna informazione viene fornita al consumatore circa l'utilizzo in sicurezza della sola parte edibile del prodotto acquistato, che quindi viene impiegato tal quale.

\section{Materiali e Metodi}

\section{Campionamento}

Nel periodo compreso tra marzo 2011 e febbraio 2012 sono stati acquistati ed esaminati 90 campioni di gamberi, di cui 45 di prodotto surgelato, e 45 di prodotto decongelato e posto in vendita sfuso. I gamberi analizzati appartengono alla superfamiglia Penaeoidea, ed in particolare sono stati oggetto dell'indagine le specie: Paeneus longirostris, Pleoticus muelleri, Penaeus kerathurus, Penaeus vannamei, Penaeus monodon, Penaeus indicus, Pandalus borealis, catturati in diverse zone FAO. I 45 campioni di prodotto surgelato includevano 15 campioni di gamberi interi con esoscheletro, 15 campioni di code di gamberi sgusciate e 15 code di gambero con esoscheletro; tutti dichiaravano in etichetta la provenienza da Paesi extraeuropei. La scelta delle confezioni di gamberi surgelati da sottoporre ad analisi è stata effettuata in modo da diversificare il più possibile la provenienza, al fine di ottenere dati significativi sull'impiego di solfiti nei Paesi extraeuropei produttori ed esportatori di gamberi e verificare se tale additivazione rispetta i limiti di impiego previsti dalla legislazione europea. I 45 campioni di gamberi decongelati oggetto dell'indagine, tutti interi e provvisti di esoscheletro, erano venduti sfusi nelle pescherie e nei mercati ittici; di questi, 25 campioni erano esposti al pubblico con la sola indicazione di prodotto decongelato e non è stato possibile recuperare dati circa la zona di provenienza, la denominazione scientifica e/o commerciale e l'eventuale aggiunta di solfiti; l'identificazione della specie di appartenenza è stata effettuata per analisi morfologica delle caratteristiche anatomiche macroscopiche. I restanti 20 campioni di prodotto decongelato, invece, sono stati acquistati presso la grande distribuzione organizzata (GD0) e per essi è stato possibile reperire tutte le indicazioni obbligatorie previste dalla legislazione per il comparto ittico, da riportare in etichetta.

Dopo l'acquisto i campioni sono stati tra- sportati in laboratorio e mantenuti alla temperatura di congelamento fino al momento dell'analisi, che è stata condotta sul prodotto tal quale e sull'esoscheletro, laddove i campioni ne fossero provvisti.La ricerca dei solfiti è stata eseguita impiegando il metodo MonierWilliams (AOAC, 1995), che separa l'anidride solforosa dalle matrici alimentari complesse, effettuando una distillazione in corrente di vapore e la determinazione quantitativa è effettuata mediante titolazione. Le analisi sono state condotte utilizzando l'apparecchio UDK132 (VELP Scientifica, Usmate, Italy), che garantisce una riproducibilità e una correlazione con il metodo Monier-Williams pari a 0,991 .

\section{Preparazione del campione}

Dieci g di campione tal quale, e $10 \mathrm{~g}$ di solo esoscheletro (per i campioni provvisti), sono stati omogenati in UltraTurrax, e posti nell'unità di distillazione.

\section{Distillazione e titolazione}

L'apparecchio di distillazione UDK132 (VELP Scientifica) è stato attivato per una distillazione della durata di $6 \mathrm{~min}$; al distillato raccolto sono stati aggiunti $75 \mathrm{~mL}$ di acqua deionizzata, $1 \mathrm{~mL}$ della soluzione indicatrice di amido, 3-4 gocce della soluzione di iodio 0,02 $\mathrm{N}$; la soluzione ottenuta di colore blu viene posta su agitatore magnetico. Non appena inizia lo sviluppo di vapori di distillazione si inizia la titolazione facendo gocciolare lentamente la soluzione di iodio $0,02 \mathrm{~N}$ nel becher. $\mathrm{Si}$ prosegue la titolazione finché non viene più consumato iodio ed il colore blu rimane invariato per $30-40 \mathrm{~s}$.

Il calcolo della concentrazione dei solfiti, espresso come anidride solforosa $\mathrm{SO}_{2}$ è dato dalla seguente equazione:

$$
\mathrm{SO}_{2}(\mathrm{ppm})=\mathrm{V}^{*} \mathrm{~N}^{*} 32 * 1000 / \mathrm{W}
$$

dove, $\mathrm{V}=\mathrm{mL}$ di soluzione $0,02 \mathrm{~N}$ di iodio standardizzata consumati, $\mathrm{N}=$ normalità esatta della soluzione di iodio utilizzata, $32=$ peso equivalente dell'anidride solforosa, $1000=$ conversione da mg mole a $\mathrm{g}$ mole, $\mathrm{W}=$ peso del campione in $\mathrm{g}$.

Tutti i campioni sono stati analizzati in doppio, e la concentrazione dei solfiti rilevati è stata espressa come media aritmetica dei due valori ottenuti.

\section{Risultati}

Dalla Tabella 1 si evince come tutti i campioni di gamberi surgelati sgusciati presentano una concentrazione residuale di $\mathrm{SO}_{2}$ inferiore a $150 \mathrm{mg} / \mathrm{kg}$ di $\mathrm{SO}_{2}$, limite disciplinato dal Regolamento UE 1129/2011 (Commissione 
Europea, 2011); la concentrazione residuale media dei campioni analizzati espressa come $\mathrm{SO}_{2} \mathrm{mg} / \mathrm{kg}[ \pm$ standard deviation (S.D.)] risulta essere pari a $112,90 \pm 27,55$.

Sia i campioni di gamberi surgelati venduti interi, sia le code di gambero surgelate provviste di esoscheletro, invece, presentano concentrazioni di solfiti superiori al limite di legge; per tali campioni la concentrazione residuale media espressa come $\mathrm{SO}_{2} \mathrm{mg} / \mathrm{kg}( \pm$ S.D. $)$ risulta essere pari a $214 \pm 17,43$ e $170,73 \pm 14,99$, rispettivamente.

La concentrazione di solfiti risulta enormemente più elevata se si analizzano i dati relativi all'esoscheletro separato dal tessuto edibile.

Dall'analisi della Tabella 2 si evince come 34 campioni di gamberi interi decongelati venduti sfusi, su 45 campioni esaminati, presentano una concentrazioni di solfiti superiori al limite imposto dalla legislazione cogente; la concentrazione residuale media espressa come $\mathrm{SO}_{2}$ $\mathrm{mg} / \mathrm{kg} \quad( \pm$ S.D. $)$ risulta essere pari a $160,05 \pm 26,15$ per i campioni acquistati presso la GD0 e 292,54 146,04 per i campioni provenienti da mercati ittici e pescherie.

Anche in questo caso l'indagine condotta anche sull'esoscheletro ha mostrato valori di solfiti particolarmente elevati.

Inoltre al fine di valutare l'efficacia della metodica impiegata in fase di recupero e la correlazione con il metodo di riferimento Monier-Williams, 10 aliquote da $10 \mathrm{~g}$ di uno stesso campione di gamberi sono state fortificate, aggiungendo quantità note di una soluzione standard di sodio metabisolfito (50 $\mathrm{mg} / \mathrm{L}$ ): il recupero medio calcolato \pm S.D. è risultato essere pari al $96,9 \pm 6,4 \%$.

\section{Discussione}

L'elevata percentuale di gamberi additivati con solfiti oltre i limiti imposti dalla normativa, evidenziata con la nostra indagine, risulta particolarmente grave per il rischio sanitario e i risvolti tossicologici acuti che il consumo di questo prodotto può causare in soggetti particolarmente sensibili. Non sono altresì da sottovalutare i risultati ottenuti sulla presenza di solfiti sull'esoscheletro; infatti qualunque sia la tipologia di vendita (surgelato $v s$ decongelato; intero $v s$ code di gambero) e la modalità con la quale è stata effettuata la pratica di additivazione (immersione, nebulizzazione, aspersione di soluzioni a base di solfiti), l'esoscheletro sequestra la maggior parte dell'additivo utilizzato e pertanto fornisce indicazioni importanti per l'ispezione sanitaria e il controllo della tecnologia di produzione.

Se si considera la provenienza dei campioni esaminati si desume che la scorretta additivazione dei solfiti non è collegata ad una specifica area di cattura/produzione extraeuropea, ma ci consente di ammettere che le pratiche di additivazione dei crostacei sono eseguite senza alcun controllo e senza rispettare la normativa vigente del Paese europeo importatore.

I punti di ispezione frontaliera (PIF), deputati al controllo delle derrate alimentari in ingresso dai Paesi terzi, come è noto, effettuano controlli mirati quasi esclusivamente alla verifica del mantenimento della catena del freddo, all'esame organolettico e documentale di trasporto.

In realtà dovrebbero essere effettuati anche i necessari controlli per la verifica del rispetto delle normative comunitarie, soprattutto in tema di additivazioni, ma sono a tutti note le problematiche che si incontrano nell'espletamento di tali controlli, determinate dal costo del mantenimento in cella del prodotto in attesa del referto analitico, dell'elevata deperibilità del prodotto e dell'importante valore commerciale.

I risultati relativi al prodotto commercializzato nella GD0, sempre molto impegnata e vigile nella qualifica dei fornitori, nel caso della commercializzazione dei gamberi, sembra non attenersi ai rigorosi protocolli interni previsti. Comparando la media delle concentrazioni di solfiti riscontrata nei prodotti venduti decongelati e sfusi dalla GD0 $\left(160,05 \pm 26,15 \mathrm{mg} / \mathrm{kg}\right.$ di $\left.\mathrm{SO}_{2}\right)$ con quella riscontrata nelle pescherie/mercati ittici $\left(292.54 \pm 146.04 \mathrm{mg} / \mathrm{kg}\right.$ di $\left.\mathrm{SO}_{2}\right)$ si evince una sostanziale differenza, che fa presupporre un ulteriore utilizzo di solfiti durante la fase di commercializzazione.

Particolare importanza sanitaria rivestono i risultati della presenza di solfiti riscontrata sui gamberi sgusciati, in quanto questo prodotto ricopre una ampia fetta di mercato, perché prontamente utilizzabile dal consumatore. L'asportazione del cefalotorace, del carapace e dell'esoscheletro addominale nella produzione secondaria è una pratica ormai molto diffusa, essendo nota la presenza del complesso enzimatico PPO su questi apparati e tessuti; tuttavia spesso anche il prodotto sgusciato viene trattato con i solfiti al fine di ottenere un prodotto meno deperibile anche all'esame organolettico. Se il consumo di tale prodotto prevede la bollitura il rischio sanitario è da considerare minimo, in quanto parte dei solfiti è disperso dal trattamento e riferibile quasi esclusivamente ai consumatori sensibili, ma se il prodotto viene consumato tal quale crudo, o cotto con modalità differenti, il rischio sanitario è più grave.

Un altro aspetto importante legato al controllo e alla vigilanza degli alimenti ed evidenziato dalla nostra indagine è stato quello di aver ancora riscontrato nei mercati ittici e in alcune pescherie prodotti senza l'indicazione precisa della provenienza o con dichiarazioni ingannevoli da parte del rivenditore.

Per ottenere, infatti, le informazioni sulla provenienza dei campioni di gamberi da noi acquistati, che non erano etichettati abbiamo interpellato i rivenditori, che hanno indicato, quale zona di pesca, il Mar Mediterraneo. Tuttavia, alcune specie in vendita non sono presenti in questo mare. Per quanto attiene, invece, la presenza di additivi, i rivenditori hanno confutato il possibile utilizzo, assicurando quindi l'assenza di sostanze allergizzanti, come i solfiti.

L'indicazione in etichetta della presenza di solfiti è resa obbligatoria dal D.Lgs 114/2006 (Repubblica Italiana, 2006), che modifica il D.Lgs 109/92 (Repubblica Italiana, 1992), e che di fatto rende obbligatorio per tutti gli addetti dei vari segmenti produttivi e di commercializzazione degli alimenti di riportare in etichetta tutti gli allergeni (e tossici), ed in particolare per l'anidride solforosa e i suoi derivati prescrive che venga riportata la dicitura contiene solfiti qualora si superino i 10 ppm nell'alimento.

Tra gli additivi alimentari, proprio i solfiti sono stati e sono oggetto di studio scrupoloso da parte della comunità scientifica in ragione del fatto che il loro impiego è sempre più spesso indiziato quale responsabile della comparsa di fenomeni di intolleranza caratterizzati da emicrania, disturbi gastrointestinali, disturbi comportamentali e reazioni allergiche in individui sensibili. Senza dubbio, sono queste ultime a destare le maggiori preoccupazioni per la possibile insorgenza di broncospasmo con esito talora letale come nei ben documentati casi di decessi, risalenti agli anni Settanta che nel 1986 hanno indotto l'FDA a revocare lo stato di generally recognized as safe (GRAS) prima attribuito ai solfiti, tenendo conto della potenziale tossicità di questi composti, e a sottoporre tali additivi ad una serie di valutazioni scientifiche, negli anni successivi, per la definizione da parte del Joint FAO/WHO Expert Committee on Food Additives dell'acceptable daily intake (ADI) stabilito in $0,7 \mathrm{mg} / \mathrm{kg}$ peso corporeo (FAO/WHO, 2007).

\section{Conclusioni}

Si auspica un maggior controllo da parte delle Autorità competenti sulla presenza dei solfiti nei crostacei e nelle altre tipologie di alimenti dove l'additivazione è consentita, in quanto il rischio sanitario è elevato e il danno tossicologico può essere anche grave.

Inoltre, sarebbe opportuno che le indicazioni in etichetta, per tutti i crostacei venduti provvisti di esoscheletro, fossero integrate con precise modalità di utilizzo da parte del consumatore consigliando di impiegare il prodotto tal quale solo dopo un adeguato risciacquo e/o bollitura o previa eliminazione dell'esoscheletro. 
Organization ed., Ginevra, Svizzera.

Fazio T, Warner CR, 1990. A review of sulphites in foods: analytical methodology and reported finding. Food Addit Contam 7:433-54

FDA, 2000. Sulfites: an important food safety issue, an update on regulatory status and methodologies. Food and Drug Administration ed., Silver Spring, MD, USA.

Garcia-Molina F, Penalver MJ, Rodriguez-Lopez JN, Garcia-Canovas F, Tudela J, 2005. Enzymatic method with polyphenol oxidase for the determination of cysteine and N-acetylcysteine. J Agr Food Chem 53:6183-9.

Haahtela T, Hannuksela M, 1989. Food additives and hypersensitivity. In: Branen AL, Davidson PM, Salminen S, eds. Food Additives. Marcel Dekker Publ., New York, NY, pp 622-623.

Hardisson A, Rubia C, Frías I, Rodríguez I, Reguera JI, 2002. Content of sulphite in frozen prawns and shrimps. Food Control 13:275-9.

Lester MR, 1995. Sulphite sensitivity: significance in human health. J Am Coll Nutr 14:229-32.

Mcevily AJ, lyengar R, Otwell S, 1991. Sulfite alternative prevents shrimp melanosis. Food Technol-Chicago 45:80-6.

Montero P, Lopez-Caballero ME, Perez-Mateos M, 2001. The effect of inhibitors and high pressure treatment to prevent melanosis and microbial growth on chilled prawns (Penaeus japonicus). J Food Sci 66:1201-6.

Otwell WS, Marshall M, 1986. Studies on the use of sulfites to control shrimp melanosis (Blackspot). University of Florida ed., Gainesville, FL, USA.

Pearson D, 1976. The chemical analysis of foods. Churchill Livingstone, Edinburgh,
Gran Bretagna.

Rotllant G, Arnau F, García JA, García N, Rodríguez M, Sardà F, 2002. Effect of metabisulphite treatments and freezing on melanosis inhibition in rose shrimp Aristeus antennatus. Food Sci Technol Int 8:243-7.

Ruiter A, Bergwerff AA, 2005. Analysis of chemical preservatives in foods. In: Ötles S, ed, Methods of analysis of food components and additives. Taylor \& Francis, Boca Raton, FL, USA.

Ruiz-Capillas C, Jiménez-Colmenero F, 2009. Application of flow injection analysis for determining sulphites in food and beverages: a review. Food Chem 112:487-93.

Taylor SL, Higle NA, Bush RK, 1986. Sulfites in foods: uses, analytical methods, residues, fate, exposure assessment, metabolism, toxicity, and hypersensitivity. Adv Food Res 30:1-76.

Vally H, Misso NL, Madan V, 2009. Clinical effects of sulphite additives. Clin Exp Allergy 39:1643-51.

Walker R, 1985. Sulphiting agents in foods: some risks/benefit considerations. Food Addit Contam 24:5.

Williams DJ, Slaterry SL, Nottingham SM, 1990. A comparison of selected methods for determining sulphite in prawns. J Food Protect 53:875-7.

Wood R, Foster L, Damant A, Key P, 2004. Analytical methods for food additives. CRC Press, Boca Raton, FL, USA.

Zamorano JP, Martinez-Alvarez 0, Montero P, Gomez-Guillen MC, 2009. Characterisation and tissue distribution of polyphenol oxidase of deepwater pink shrimp (Parapenaeus longirostris). Food Chem 112:104-11. 